Ein fach- und paradigmenübergreifendes Modell der Forschungskompetenz in den Sozialwissenschaften

EN: Model of research competencies spanning across social scientific disciplines and research paradigms

\title{
Christopher Gess*
}

Humboldt-Universität zu Berlin

Institut für Erziehungswissenschaften

Unter den Linden 6

10099 Berlin

Email: gesschri@hu-berlin.de

Tel. ++491717096021

\section{Julia Rueß}

Humboldt-Universität zu Berlin

bologna.lab

Unter den Linden 6

10099 Berlin

Email: ruessjul@hu-berlin.de

Tel. ++4930209370811

\section{Sigrid Blömeke}

University of Oslo/ Faculty of Education

Centre for Educational Measurement

Niels Henrik Abels hus

$\mathrm{N}-0318$ Oslo, Norway

Email: sigribl@cemo.uio.no

Tel. ++4746418755

* korrespondierender Autor 


\section{Zusammenfassung (DE)}

Obwohl die Befähigung zu eigenständiger Forschung ein zentrales Ziel des Studiums ist, fehlt es in den Sozialwissenschaften an einem theoretischen Modell von Forschungskompetenz. In der vorliegenden Arbeit wurde ein fach- und paradigmenübergreifendes Modell sozialwissenschaftlicher Forschungskompetenz in einem sequenziellen Mixed-Methods-Design entwickelt: Mittels Experteninterviews ( $n=4)$ wurden erforderliche Kompetenzdimensionen identifiziert und mittels schriftlicher Expertenbefragung $(n=8)$ ausdifferenziert. Die Bedeutsamkeit der Modellbestandteile wurde über ein Expertenrating ( $n=80)$ untersucht. Im Ergebnis konnte ein Modell mit den Kompetenzdimensionen Forschungsprozess-, Methoden- und methodologisches Wissen abgeleitet werden. Diese Dimensionen sind für die Forschungstätigkeiten der Problemherleitung, Forschungsplanung und Datenauswertung und -interpretation entscheidend.

Schlagwörter: Forschungskompetenz, Methodenwissen, Kompetenzmodellierung, Testentwicklung, Praxisexperten, Sozialwissenschaften

\section{Abstract (EN)}

Although the development of research competency is an important goal of higher education in social sciences, there is no theoretical model of social-scientific research competency. In the present study, a model spanning across social-scientific disciplines and research paradigms was developed: Relevant competency dimensions were identified through expert interviews $(n=4)$. Their roles in performing key research steps were described based on an expert survey $(n=8)$. Finally, an expert rating $(n=80)$ was performed to determine the importance of each aspect of the competency model. The resulting model entailed the dimensions research process knowledge, knowledge of research methods, and knowledge of methodologies as well as the research steps finding and defining a research problem, planning a research project, and analysing and interpreting data.

Keywords: Research competency, research methods education, competency modelling, test construction, vocational experts, social sciences 


\section{Einleitung}

Kompetenzorientierung wird in der hochschulischen Lehre seit Langem gefordert (Wild \& Esdar, 2016). Die Studienprozesse und die zugehörige Lehr- und Lernforschung sollen auf die Aneignung von Kompetenzen ausgerichtet sein (Wissenschaftsrat, 2008). Trotz intensiver Bemühungen (bspw. im Forschungsprogramm Kompetenzmodellierung und Kompetenzerfassung im Hochschulsektor) bestehen hier noch größere Forschungslücken (ZlatkinTroitschanskaia, Shavelson, \& Kuhn, 2015).

Wenig fortgeschritten ist die Kompetenzorientierung der Lehr- und Lernforschung zur sozialwissenschaftlichen Forschungsausbildung. In Übersichtsstudien wurden zwei zentrale Defizite dieses Forschungsfeldes herausgearbeitet: es mangelt erstens an empirischen Studien zu Lehrmethoden und Lernprozessen jenseits anekdotischer Evidenzen (Kilburn, Nind, \& Wiles, 2014) und zweitens an Diskussionen „of how we assess students, [and] of what the learning objectives of an introductory research methods course should be“ (Earley, 2014). Dies ist überraschend, da der Erwerb von Forschungskompetenz ein grundlegendes Ziel hochschulischer Bildung markiert (Wissenschaftsrat, 2006) und zudem in verschiedenen sozialwissenschaftlichen Tätigkeitsfeldern als Qualifizierungsanforderung fest verankert ist (bspw. Deutsche Gesellschaft für Evaluation, 2008; Esser, Groeger, Munz, Lehndorfer, \& Schwartz, 2010; European Commission, 2013).

Im vorliegenden Beitrag soll diese Forschungslücke teilweise geschlossen werden, indem ein Modell der kognitiven Facette von Forschungskompetenz in den Sozialwissenschaften entwickelt wird. Forschungskompetenz wird dabei in Anlehnung an Klieme und Leutner (2006) verstanden als erlernbare, kognitive Leistungsdisposition, die sich funktional auf Situationen und Anforderungen der empirischen Sozialforschung bezieht und somit über die Rezeption von Forschung (Groß Ophoff, Schladitz, Lohrmann, \& Wirtz, 2014) hinausgeht. Da Studien zur Forschungsausbildung aus interdisziplinärer Perspektive mehrerer sozialwissenschaftlicher Studienfächer mangeln (Kilburn et al., 2014), soll mit einem fachübergreifenden Modell die Grundlage für solche Perspektiven gelegt werden. Es werden jene Studienfächer einbezogen, die sich ihren Forschungsgegenständen mit Methoden der empirischen Sozialforschung nähern. Paradigmenübergreifend wird der Fokus auf die empirische Sozialforschung gelegt. Zwar ist damit eine Eingrenzung verbunden, indem die theoretische und geisteswissenschaftlich orientierte Forschung nicht betrachtet wird. Gleichwohl wird die gesamte Breite empirischer Methoden einbezogen, d.h. sowohl quantitativ-statistische als auch qualitativ-interpretative bzw. -rekonstruktive Verfahren berücksichtigt.

\subsection{Modelle von Forschungskompetenz}

Es gibt bislang keinen wissenschaftlichen Diskurs zur Struktur sozialwissenschaftlicher Forschungskompetenz (Earley, 2014). Die vereinzelt vorliegenden Modelle beziehen sich auf 
unterschiedliche Kontexte: (1) generische Modelle, (2) Modelle im Kontext der Lehrerbildung und schließlich (3) Modelle, die auf Professionen mit Psychologiebezug begrenzt sind. Im Folgenden soll ein erster Versuch unternommen werden, die unterschiedlichen Perspektiven zusammenzuführen.

\subsubsection{Generische Forschungskompetenz}

Thiel und Böttcher (2014) haben ein Kompetenzstrukturmodell entwickelt, das für alle Studienfächer anwendbar sein soll. Die fachübergreifende Ebene des Modells wird entlang des Forschungsprozesses in vier Kompetenzdimensionen aufgeteilt: Recherche-, Methoden-, Kommunikations- und Reflexionskompetenz. Die fachspezifische Ebene des Modells wird untergliedert in die Kenntnis fachlicher Theorien, Methoden, aktueller Befunde und Kommunikationsstandards. Eine Beschreibung dieser Kompetenzdimensionen wird jedoch nicht vorgenommen, was vermutlich dem Anspruch geschuldet ist, ein für alle Fächer gültiges Modell zu entwickeln: Die Methodenkenntnis etwa wird in der Erziehungswissenschaft ein anderes Konstrukt sein als in der Physik.

Im Unterschied zu diesem Ansatz ist der vorliegende Beitrag darauf ausgerichtet, ein Modell für eine Fächergruppe mit weitgehend einheitlichen Forschungsmethoden zu entwickeln, so dass die jeweiligen Kompetenzdimensionen inhaltlich beschrieben werden können.

\subsubsection{Auf die Lehrerbildung begrenzte professionsspezifische Modelle}

In Lehramtsstudiengängen gewinnt die Forschungskompetenz im Zuge der Einführung des Praxissemesters und damit verbundenen studentischen Forschungsarbeiten zunehmend an Bedeutung. In den Bildungsstandards der Lehrerbildung (Kultusministerkonferenz, 2014) werden Forschungskompetenzen indirekt über die Selbst- und Fremdevaluation angesprochen, indem die Vermittlung von Kenntnissen über Methoden, Verfahren und Instrumente der Evaluation gefordert wird.

Meyer (2003) hat ein Kompetenzmodell auf Basis eigener Lehrerfahrungen entwickelt. Es sieht je drei kognitive, affektiv-motivationale und „pragmatisch-handlungsbezogene“ Kompetenzdimensionen vor (Meyer, 2003, S. 109). Als kognitive Dimensionen werden das Erlernen der Wissenschaftssprache, die Einsicht in die Mehrperspektivität von Forschung und Begrenztheit des eigenen Forschungshorizonts sowie die Einsicht in die Komplexität des Theorie-Praxis-Verhältnisses berücksichtigt. In der pragmatisch-handlungsbezogenen Facette wird mit der forschungsbezogenen Methodenkompetenz eine weitere kognitive Dimension angeführt.

Ivanenko et al. (2015) haben allgemeine Kompetenzmodelle für Lehramtsstudierende analysiert und Bestandteile mit Forschungsbezug in ein Modell von Forschungskompetenz überführt. Es enthält zwei kognitive Kompetenzdimensionen. Die methodologisch-reflexive 
Dimension wird anhand von Performanzaussagen zu mehreren Forschungstätigkeiten beschrieben, bspw. die „ability to (...) determine methods, means and sequence of steps“ (Ivanenko et al., 2015, S. 226). Die tätigkeitsbezogene Dimension beinhaltet die Kommunikation und Anwendung von Forschungsergebnissen. Zum Teil werden innerhalb der Dimensionen allerdings sehr unterschiedliche Subdimensionen zusammengefasst.

Zwischen den beiden Modellen findet sich bei den kognitiven Kompetenzdimensionen nur eine inhaltliche Überscheidung: die Kenntnis sozialwissenschaftlicher Forschungsmethoden. Sie wird jedoch jeweils nicht näher beschrieben. Dieses Defizit soll mit dem vorliegenden Beitrag adressiert werden.

\subsubsection{Auf Professionen mit Psychologiebezug begrenzte Modelle}

Weitere Modelle wurden für Professionen mit Psychologiebezug entwickelt. Für das Tätigkeitsfeld des occupational therapists, das in den USA ein Studium mit psychologischen Anteilen voraussetzt, wurde ein Bewertungsraster von beruflichen Forschungsleistungen entwickelt (Abreu, Peloquin, \& Ottenbacher, 1997). Es enthält vier Dimensionen: Das Methodenwissen soll dazu beitragen, methodische Prozeduren korrekt auszuwählen und anzuwenden. Das organisationale Wissen umfasst die Kenntnis von Regeln, ethischen Prinzipien und Machtverhältnissen im Arbeitskontext. Der dritte Bereich kann als Wertschätzung der Bedeutung von Forschung für die Praxis interpretiert werden. Viertens wird Wissen zum Forschungsgegenstand genannt.

Ein weiteres Modell liegt für das Tätigkeitsfeld des counselors vor (Wester \& Borders, 2014). Auf Basis einer Delphi-Studie mit 15 ForschungsexpertInnen identifizieren Wester und Borders eine Liste von 159 kleinteiligen Forschungskompetenzen in sechs Dimensionen: kritisches Denken, Forschungsschritte, ethische Kompetenzen, Würdigung des Umfangs von Forschung, Sozialkompetenz und Neigung zur Weiterbildung.

Mit seinen 159 kleinteiligen Kompetenzen beinhaltet das Modell für counselors auch Teile von drei der vier Kompetenzdimensionen des Modells für occupational therapists. Auf der konzeptionellen Ebene übergeordneter Kompetenzdimensionen weisen die beiden Modelle jedoch keine Gemeinsamkeiten auf.

\subsection{Desiderate und Forschungsfragen}

Aus der Betrachtung vorliegender Kompetenzmodelle lassen sich zusammenfassend folgende Desiderate erkennen: Erstens fehlt ein für die Sozialwissenschaften fachübergreifendes Kompetenzmodell. Aus den vorliegenden Modellen lassen sich mangels Gemeinsamkeiten (mit Ausnahme der Methodenkompetenz) keine Aussagen zu Dimensionen von Forschungskompetenz ableiten. Diesem Desiderat widmet sich der vorliegende Beitrag: Welche Dimensionen von sozialwissenschaftlicher Forschungskompetenz lassen sich identifizieren? 
Zweitens sind die meisten vorliegenden Modelle stark tätigkeitsbezogen entlang des Forschungsprozesses konzipiert. Vielfach führt dies zu einer Operationalisierung in Form von Performanzaussagen zu Forschungstätigkeiten, das heißt zu situationsspezifischen Komponenten von Forschungskompetenz. Solche Performanzaussagen lassen keine Rückschlüsse über zugrundeliegende personenspezifische Komponenten der Forschungskompetenz zu. Eine zentrale Herausforderung der Kompetenzmodellierung ist es, zwischen personen- und situationsspezifischen Komponenten zu differenzieren und sie aufeinander zu beziehen (Klieme \& Leutner, 2006). Daraus leitet sich folgende Forschungsfrage für den vorliegenden Beitrag ab: Welche Kompetenzdimensionen liegen welchen Forschungstätigkeiten zugrunde?

Drittens wurden Personen aus der beruflichen Praxis nicht systematisch in die Modellierungsbemühungen einbezogen - auch nicht bei den professionsspezifischen Modellen. Da sich der Kompetenzbegriff unter anderem auf die Bewältigung beruflicher Anforderungen bezieht (Weinert, 2001a), lässt sich folgende Frage ableiten: Welche Dimensionen des Modells werden auch von PraxisexpertInnen als bedeutsam angesehen?

\section{Methodisches Vorgehen}

In Anlehnung an die identifizierten Desiderate und vorliegende Modelle baut die Grundstruktur des zu entwickelnden Kompetenzstrukturmodells auf zwei Achsen auf. Auf der ersten Achse werden - zu identifizierende - Kompetenzdimensionen abgebildet (personenspezifische Komponenten). Auf der zweiten Achse werden, orientiert an vorliegenden Modellen, die häufig über eine Auflistung von Forschungsschritten operationalisiert sind, Forschungstätigkeiten abgebildet (situationsspezifische Komponenten). Beide Achsen werden aufeinander bezogen, indem das Modell beschreibt, inwiefern die Kompetenzdimensionen den Forschungstätigkeiten zugrunde liegen.

Aufbauend auf dieser Grundstruktur mit zwei Achsen wurde die Kompetenzmodellierung anhand eines Mixed-Methods Designs vorgenommen. Genutzt wurde ein sequenzielles Verallgemeinerungsdesign (Kuckartz, 2014, S. 81), das zunächst mit qualitativen Methoden auf Exploration ausgelegt ist und die qualitativen Befunde mittels quantitativer Methoden zu verallgemeinern sucht. Das Vorgehen zeichnet sich durch eine zu Beginn explorative Betrachtung von Forschungskompetenz und eine zunehmend wachsende Verengung der methodischen Perspektive aus. Die relevanten Kompetenzdimensionen und Forschungstätigkeiten wurden in Experteninterviews identifiziert und im Anschluss inhaltsanalytisch über eine schriftliche Expertenbefragung ausdifferenziert. Abschließend wurde eine Expertenbegutachtung durch Praxis- und universitäre ExpertInnen durchgeführt.

\subsection{Schritt 1: Experteninterviews}


Sampling. Es wurden vier ExpertInnen für sozialwissenschaftliche Forschung interviewt. Sie sollten über umfangreiche Erfahrung mit forschenden Studierende verfügen (etwa aus Forschungsseminaren und -werkstätten) und zudem unterschiedliche Methodentraditionen vertreten (siehe Tab. 1). Zudem sollten sie nachweisbare Methodenexpertise aufweisen, d.h. eine Methodenveranstaltung angeboten oder ein -lehrbuch veröffentlicht haben.

Erhebung. Wie für theoriegenerierende Experteninterviews empfohlen (Meuser \& Nagel, 2005), wurde die Interviewsituation als offenes Gespräch angelegt. Zunächst wurden zur Identifizierung von Kompetenzdimensionen primär erzählgenerierende Fragen gestellt (z.B. „Können Sie mir von einer Studierenden erzählen, deren Forschungsfähigkeit sich stark entwickelt hat?"). Zur Bestimmung zentraler Forschungstätigkeiten wurden die ExpertInnen mit verschiedenen Forschungstätigkeiten konfrontiert und gebeten, deren Bedeutung in ihrer Methodentradition zu beschreiben. Die Interviews dauerten zwischen 45 und 90 Minuten.

Auswertung. Zur Auswertung der Interviewtranskripte wurde auf die Grounded Theory (Charmaz, 2014) zurückgegriffen. Es wurden zumeist ganze Aussagen mit mehreren Sätzen als Analyseeinheit kodiert. Insgesamt wurden 271 Kodierungen in 112 textnah formulierten Kodes vorgenommen. Da die ExpertInnen selbst Begriffe auf hohem Abstraktionsniveau nutzten, waren die Kodes teilweise bereits stark analytisch. Sie wurden thematisch zu Kategorien gruppiert und Kernkategorien und deren Beziehungen untereinander herausgearbeitet.

Tabelle 1: $\quad$ Übersicht zu den interviewten ExpertInnen

\begin{tabular}{|c|c|c|c|c|}
\hline Methodologie & Kennung & Fach & 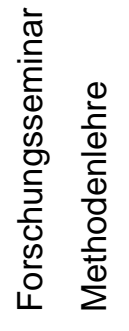 & 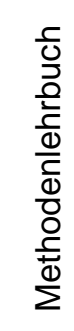 \\
\hline Vergleichende Politikwissenschaft & QL1.I & Politikwissenschaft & $\checkmark \checkmark$ & \\
\hline Ethnografie & $\mathrm{QL} 2 \cdot \mathrm{I}$ & Erziehungswissenschaft & $\checkmark \checkmark$ & \\
\hline Dokumentarische Methode & QL3.I & Erziehungswissenschaft & $\checkmark$ & $\checkmark$ \\
\hline Quantitative Sozialforschung & QN1.I & Soziologie & $\checkmark$ & \\
\hline
\end{tabular}

Anmerkungen: Forschungsseminar $=$ Leitung einer Forschungswerkstatt, eines -seminars oder -kolloquiums. QN = quantitativ ausgerichtete/r Expertln, $\mathrm{QL}=$ qualitative/r Expertln, I = Interview, $\mathrm{B}=$ Befragung.

\subsection{Schritt 2: Expertenbefragung}

Sampling. Bei der schriftlichen Expertenbefragung wurde gegenüber den Experteninterviews als zusätzliches Auswahlkriterium die Vorstandstätigkeit in der Methodensektion einer 
sozialwissenschaftlichen Fachgesellschaft berücksichtigt. An dieser schriftlichen Befragung nahmen 4 qualitative und 4 quantitative MethodenexpertInnen teil (siehe Tab. 2).

Erhebung. Die schriftliche Expertenbefragung baute auf den Ergebnissen des ersten Arbeitsschritts auf. In offenen Textfeldern wurden die ExpertInnen gebeten, Fragen zu zuvor identifizierten Kompetenzdimensionen und Forschungstätigkeiten zu beantworten. Die ExpertInnen wurden gebeten, typische Probleme von Studierenden zu benennen und Handlungsempfehlungen wiederzugeben.

Auswertung. Mit den offenen Textfeldantworten aus der schriftlichen Expertenbefragung wurde eine zusammenfassende Inhaltsanalyse (Mayring, 2010) vorgenommen. Die Aussagen der ExpertInnen wurden den Kompetenzdimensionen und Forschungstätigkeiten durch zwei unabhängige Rater mit guter Übereinstimmung (Cohens k von 0.86, siehe Cohen, 1960) zugeordnet. Für jede Kombination aus Kompetenzdimension und Forschungstätigkeit entstand so eine Sammlung an Expertenaussagen. Die Aussagen wurden anschließend paraphrasiert und thematisch gebündelt.

Tabelle 2: $\quad$ Übersicht zu den befragten ExpertInnen

\begin{tabular}{|c|c|c|c|c|c|c|}
\hline Methodologie & Kennung & Fach & 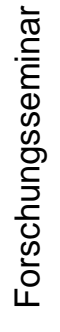 & 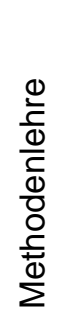 & 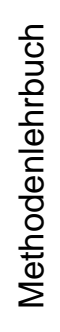 & 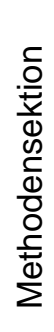 \\
\hline Dokumentarische Methode & QL3.B & Erziehungswissenschaft & $\checkmark$ & & $\checkmark$ & \\
\hline Quantitative Sozialforschung & QN1.B & Soziologie & $\checkmark$ & $\checkmark$ & & \\
\hline Quantitative Sozialforschung & QN2.B & Politikwissenschaft & & $\checkmark$ & $\sqrt{ }$ & $\checkmark$ \\
\hline Rekonstruktive Sozialforschung & QL4.B & Erziehungswissenschaft & $\checkmark$ & & & $\checkmark$ \\
\hline Dokumentarische Methode & QL5.B & Erziehungswissenschaft & $\checkmark$ & $\checkmark$ & $\checkmark$ & $\checkmark$ \\
\hline Quantitative Sozialforschung & QN3.B & Politikwissenschaft & $\checkmark$ & $\checkmark$ & $\checkmark$ & $\checkmark$ \\
\hline Rekonstruktive Sozialforschung & QL6.B & Sozialarbeit & & $\checkmark$ & & $\checkmark$ \\
\hline Quantitative Sozialforschung & QN4.B & Erziehungswissenschaft & $\checkmark$ & & & $\checkmark$ \\
\hline
\end{tabular}

Anmerkungen: Forschungsseminar $=$ Leitung einer Forschungswerkstatt, eines - seminars oder -kolloquiums. QN = quantitativ ausgerichtete/r ExpertIn, $\mathrm{QL}=$ qualitative/r ExpertIn, I = Interview, B = Befragung. . Methodensektion $=$ Vorstandsmitglied einer Methodensektion der Fachgesellschaften oder in einem ähnlichen Gremium.

\subsection{Schritt 3: Expertenrating}

Ansatz. Die Daten des Expertenratings wurden genutzt, um die Bedeutung der Bestandteile des entwickelten Kompetenzmodells zu ermitteln. Entsprechend wurde ein indirekter Zugang gewählt: Das im zweiten Schritt ausdifferenzierte Modell wurde zunächst deduktiv in 54 Kompetenztestaufgaben umgesetzt, indem für jeden zuvor identifizierten Indikator der 
Forschungskompetenz Testaufgaben entwickelt wurden (siehe Electronic Supplementary Material). Für jede Kombination aus Kompetenzdimension und Forschungstätigkeit wurden sechs Testaufgaben entwickelt. Diese Aufgaben wurden anschließend von PraxisexpertInnen hinsichtlich ihrer Relevanz für prototypische berufliche Tätigkeitsfelder und von universitären ExpertInnen hinsichtlich ihrer Eignung zur Messung von Forschungskompetenz beurteilt. Die durchschnittliche Bewertung der Testaufgaben zu einer Kompetenzdimension wurde als Indikator für die Bedeutung der zugrundeliegenden Kompetenzdimension interpretiert. Dieses indirekte Vorgehen ist einer direkten Bewertung der Kompetenzdimension vorzuziehen: Bei einer direkten Bewertung würden die ExpertInnen ihre subjektiven mentalen Repräsentation der Kompetenzdimension bewerten, nicht jedoch deren tatsächliche Operationalisierung.

Stichprobe. Die Testaufgaben wurden von PraxisexpertInnen $(n=56)$ und universitären ExpertInnen ( $n=24$ ) bewertet. Als PraxisexpertInnen wurden Sozial- und Marktforscher sowie Evaluatoren befragt. Ihre Tätigkeitsfelder setzen sozialwissenschaftliche Forschungskompetenz voraus (Engel, 2002). Alle PraxisexpertInnen gaben an, im Beruf empirische Methoden der Sozialforschung zu nutzen (siehe Tab. 3). Sie verfügen im Durchschnitt über 13.1 Jahre Berufserfahrung ( $S D=7.6)$. Je die Hälfte der universitären ExpertInnen führte im akademischen Jahr 2014/15 Methodenlehre durch oder leitete im selben Zeitraum von der Deutschen Forschungsgemeinschaft (DFG), dem Bundesministerium für Bildung und Forschung oder der Europäischen Union geförderte Projekte. ${ }^{1}$ Sie wiesen im Durchschnitt 18.4 Jahre Erfahrung $(S D=10.6)$ in Forschung und Lehre auf und hatten 21.6 Publikationen veröffentlicht $(S D=19.7)$. Die ExpertInnen decken wie in den vorangegangenen Schritten die Fächer Soziologie, Politikwissenschaft und Erziehungswissenschaft ab. Zudem wurde im dritten Schritt erstmals auch die Perspektive der Psychologie einbezogen, um eine spätere Anwendbarkeit des Tests auch in der Psychologie zu prüfen, die mitunter auch als sozialwissenschaftliche Teildisziplin gefasst wird (DFG, 2017).

Tabelle 3: $\quad$ Übersicht über den beruflichen Hintergrund und den methodischen Schwerpunkt der PraxisexpertInnen ( $\mathrm{N}=56)$

\begin{tabular}{|c|c|c|c|}
\hline Berufszweig & $\begin{array}{l}\text { ExpertIn- } \\
\text { nen }\end{array}$ & Methodologischer Schwerpunkt & $\begin{array}{l}\text { ExpertIn- } \\
\text { nen }\end{array}$ \\
\hline Evaluation & 25 & qualitative Methoden & 12 \\
\hline Marktforschung & 14 & quantitative Methoden & 12 \\
\hline Sozialforschung & 11 & sowohl qualitativ als auch quantitativ & 32 \\
\hline Beratung & 6 & & \\
\hline
\end{tabular}

${ }^{1}$ Es wurden zwei qualitative Forscher in der Psychologie ohne ein entsprechenden Forschungsprojekt befragt, da keine qualitativ forschenden Psychologen gefunden werden konnten, die dieses Kriterium erfüllten. 
Erhebung. Jede der 54 Testaufgaben wurde blockweise von 14 PraxisexpertInnen und 6 universitären ExpertInnen bewertet. Die PraxisexpertInnen wurden gebeten, die Relevanz der Inhalte der Testaufgaben im Berufsalltag auf einer Skala von 1 („gar nicht“) bis 4 („,voll und ganz") zu beurteilen. Auf derselben Skala beurteilten die universitären ExpertInnen in Anlehnung an Jenßen, Dunekacke und Blömeke (2015) die allgemeine Eignung der Testaufgaben. Im Folgenden wird dieser Vorgang in beiden Fällen abkürzend als Bewertung von Testaufgaben bezeichnet.

Auswertung. Die interne Konsistenz der Ratings wurde vorab nach Aufgabenblöcken betrachtet und lag zwischen Cronbachs a (Cronbach, 1951) von .59 bis .80 bzw. zwischen McDonalds $\omega_{t}$ (McDonald, 1999) von .80 bis .89 (siehe Tab. 4). Über Aufgabenblöcke hinweg kann die interne Konsistenz nicht berechnet werden, da den Testpersonen stets nur ein Aufgabenblock vorgelegt wurde. Zur Auswertung der Expertenbeurteilung empfehlen Jenßen et al. (2015), den arithmetischen Mittelwert zu betrachten, da dieser ein konservatives Kriterium der durchschnittlichen Einschätzung darstelle und „durch eine Gleichgewichtung aller Werte alle Experten gleichmäßig [berücksichtigt]“ (Jenßen et al., 2015: 22). Als Grenzwerte für den Ausschluss von Items empfehlen sie die arithmetische Mitte der Skala, d.h. einen Wert von 2.5. In Anlehnung an dieses Vorgehen wurden in der vorliegenden Arbeit für jede Kombination aus Kompetenzdimension und Forschungstätigkeit die Mittelwerte der Bewertungen von Testaufgaben betrachtet, um die Bedeutung der Bestandteile des Kompetenzmodells zu untersuchen. Lagen diese Mittelwerte signifikant über 2.5, wurde dies als Indikator für die Bedeutsamkeit des jeweiligen Modellbestandteils gewertet. Um dies zu prüfen, wurde ein Einstichproben-t-Test mit dem Vergleichswert 2.5 durchgeführt. Es ist gängige Praxis, parametrische Verfahren auch bei Daten, die mit Likert-Skalen erhoben wurden, anzuwenden (Norman, 2010). Dabei wurden cluster-robuste Standardfehler genutzt, da angenommen werden muss, dass alle Bewertungen eines/r Befragten voneinander abhängig sind. Aufgrund der geringeren Anzahl an Ratings durch universitäre ExpertInnen, ist der Standardfehler und somit das Konfidenzintervall dort deutlich größer.

Tabelle 4: $\quad$ Interne Konsistenz nach Aufgabenblöcken ( $\mathrm{N}=56$ PraxisexpertInnen)

\begin{tabular}{|c|c|c|}
\hline Aufgabenblock & $a$ & $\omega_{\mathrm{t}}$ \\
\hline I & .74 & .87 \\
\hline II & .67 & .80 \\
\hline III & .80 & 89 \\
\hline IV & .59 & .80 \\
\hline
\end{tabular}


Entsprechend der ersten beiden Forschungsfragen wurden Kompetenzdimensionen identifiziert und zentrale Forschungstätigkeiten bestimmt. Als Kompetenzdimensionen wurden das (1) Forschungsprozesswissen, (2) Methodenwissen und (3) methodologische Wissen herausgearbeitet. Um ein fachübergreifendes Modell entwickeln zu können, wurde die Dimension des Wissens zum Forschungsgegenstand nicht betrachtet, da sie zwingend domänenspezifisch ausgeprägt ist. Bei den Forschungstätigkeiten wiederum betonten die ExpertInnen die Bedeutung der (i) Problemherleitung, (ii) Forschungsplanung und (iii) Datenauswertung und -interpretation. Bei diesen Schritten, die auch in vorliegenden Modellen (Abreu et al., 1997; Ivanenko et al., 2015; Wester \& Borders, 2014) betrachtet werden, ließe sich am besten erkennen, wie gut Studierende forschen können.

Im Folgenden werden die Ergebnisse in drei Abschnitten berichtet, die sich an den Kompetenzdimensionen orientieren. Eine Übersicht über das finale Kompetenzmodell findet sich in Abbildung 1.
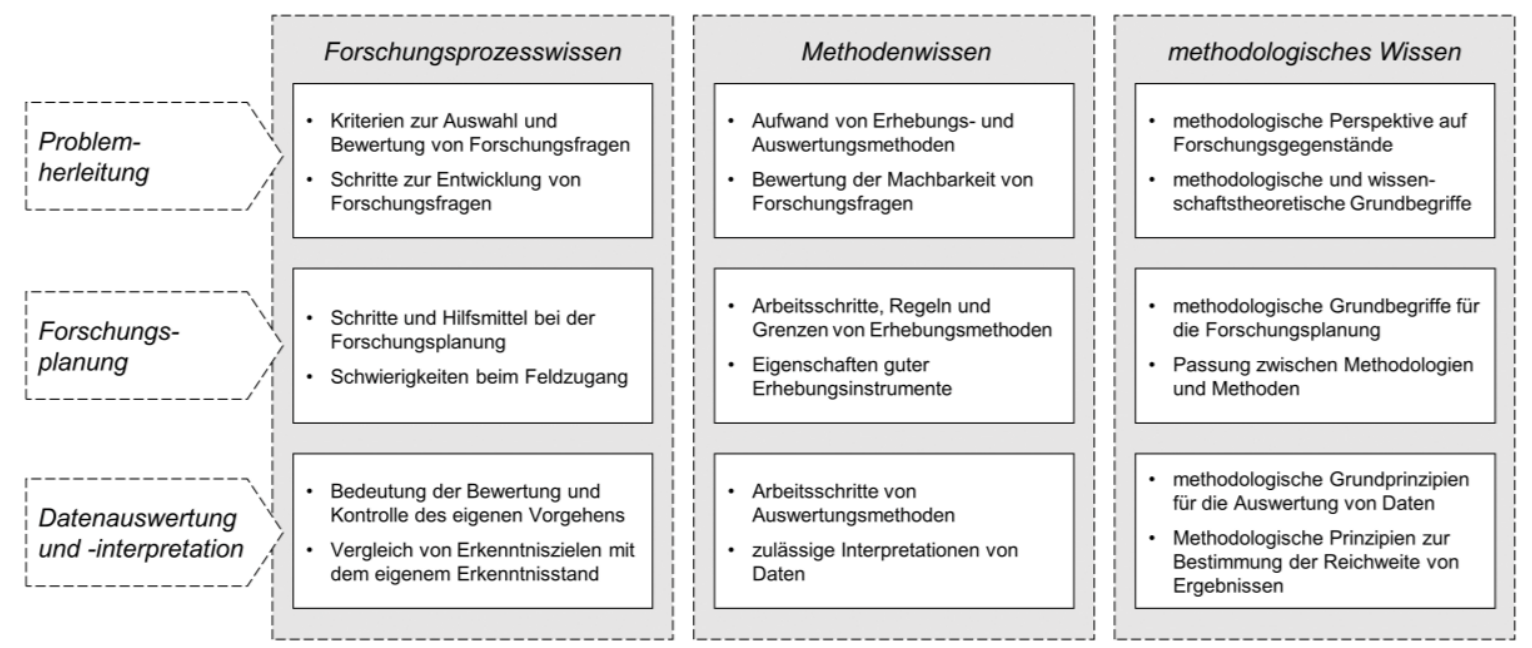

Abbildung 1: Kompetenzmodell bestehend aus drei Kompetenzdimensionen (Spalten) und drei zentralen Forschungstätigkeiten (Zeilen)

\subsection{Forschungsprozesswissen}

Das Forschungsprozesswissen ist die Grundlage für die Planung und Steuerung des eigenen Vorgehens im Forschungsprozess. Es enthält erstens das Wissen über den Ablauf von Forschung. Dazu gehört das Verständnis, welche Schritte ein wissenschaftliches Forschungsprojekt durchläuft, bspw. „wie kann ich eine Frage so formulieren, dass ich sie hinterher beforschen kann?" (QL1.I: 684). Es ist besonders in der Konzeptionsphase eines Forschungsprojekts handlungsleitend und dient unter anderem dazu, andere Kompetenzdimensionen aufzurufen: „wo kann ich mich methodisch bedienen?“ (QL1.I: 684). 
Zweitens enthält das Forschungsprozesswissen eine metakognitive Komponente. Sie umfasst das Bewusstsein der Studierenden für die Bedeutung, den eigenen Erkenntnisprozess fortwährend zu prüfen und zu steuern: „[Es] muss immer wieder neu überlegt werden: okay, wo stehen wir jetzt, [...] wohin müssen wir noch“ (QL3.I: 220). Es sind hier Strategien gemeint, die retrospektiv eine Bewertung des bisherigen Vorgehens vornehmen und prospektiv neue Planungsschritte anstoßen.

Bei der Problemherleitung ist es wichtig, dass den Studierenden die Kriterien für eine geeignete Forschungsfrage und Vorgehensweisen, eine solche zu finden, bekannt sind. Die beiden wichtigsten Schritte seien die Suche nach eigenen Forschungsinteressen („Welche Veranstaltungen waren besonders interessant?", QL6.B: 6) und die Prüfung der Umsetzbarkeit in methodischer („dass die Dinge, die benötigt werden, erhebbar sind“, QN3.B: 11) und zeitökonomischer Hinsicht („was ist im gegebenen Zeitrahmen erheb- und auswertbar?“, QL4.B: 10). Die zugehörigen Testaufgaben wurden von PraxisexpertInnen im Durchschnitt mit $2.67\left(\mathrm{Cl}_{95 \%}=[2.46 ; 2.87]\right)$ bewertet. Universitäre ExpertInnen bewerteten sie mit 2.75 $\left(\mathrm{Cl}_{95 \%}=[2.45 ; 3.05]\right)$. In beiden Expertengruppen liegt somit keine signifikant positive Bewertung vor.

Bei der Forschungsplanung ist das Wissen über Planungsschritte und -hilfsmittel sowie über Schwierigkeiten beim Feldzugang erforderlich. Zentral sei das Anlegen eines Zeitplans (QN2.B: 39, QL5.B: 10, QN3.B: 8, QL6.B: 8) und der Austausch mit anderen Studierenden (QL3.B: 45). Darüber hinaus spiele die Planung des Feldzugangs vor dem Hintergrund der Teilnahmebereitschaft (QL3.B: 42) und ethischer Fragen (QL5.B: 12) eine große Rolle. Die zugehörigen Testaufgaben wurden von beiden Expertengruppen signifikant positiv beurteilt: von PraxisexpertInnen mit $3.06\left(\mathrm{Cl}_{95 \%}=[2.87 ; 3.25]\right)$ und von universitären ExpertInnen mit $2.89\left(\mathrm{Cl}_{95 \%}=[2.57 ; 3.21]\right)$.

Bei der Datenauswertung und -interpretation ist das Forschungsprozesswissen vornehmlich metakognitiver Natur. Die steuernde Funktion des Forschungsprozesswissens wird hier besonders deutlich. So sollten Studierende ihr eigenes Vorgehen als „rekonstruktive[n] Prozess der eigenen Forschungserfahrungen darstell[en]“ (QL5.B: 17)und ihren Erkenntnisprozess kontrollieren (QL5.B: 14). Wiederkehrend wurde gefordert, dass die Studierenden ihre Ergebnisse mit Dritten diskutieren, um mögliche Probleme ihrer Interpretationen zu erkennen (QL3.B: 10, QN3.B: 42). Die zugehörigen Testaufgaben wurden von PraxisexpertInnen im Durchschnitt mit $3.08\left(\mathrm{Cl}_{95 \%}=[2.89 ; 3.28]\right)$ und von universitären ExpertInnen mit 3.19 $\left(\mathrm{Cl}_{95 \%}=[2.92 ; 3.47]\right)$ und somit signifikant positiv bewertet.

\subsection{Methodenwissen}


Das Methodenwissen hat die Funktion, die korrekte Anwendung von Forschungsmethoden sicherzustellen. Es hat eine wichtige deklarative Komponente. So betonen die ExpertInnen, dass den Studierenden „ganz klare [auswertungsmethodische] Arbeitsschritte, die sie durchlaufen sollen, mit ganz klaren Linien und Schritten“ (QL3.I: 619) vermittelt werden müssten.

Das prozedurale methodische Wissen baut auf der deklarativen Komponente auf. Es könne „nur im Forschen [er]lernt“ (QL2.I: 964) werden. Nach dem Erwerb des deklarativen Wissens „versuchen wir das mal selber zu tun“ (QL2.I: 997). Mit zunehmender Erfahrung würde eine „intuitive Kompetenz“ (QL3.I: 591, 618) bzw. eine „Praktik“ (QL2.I: 1077, 1224) entstehen. Die prozedurale Komponente wurde besonders von ExpertInnen für qualitative Methoden betont.

Bei der Problemherleitung ist das Methodenwissen zur Beurteilung des methodischen Aufwands von Forschungsfragen erforderlich. So werde der Aufwand der Auswertung qualitativer Daten (QL5.B: 12) und der Entwicklung von standardisierten Erhebungsinstrumenten (QN1.B: 2) häufig unterschätzt. Die zugehörigen Testaufgaben wurden von PraxisexpertInnen im Durchschnitt mit $2.70\left(\mathrm{Cl}_{95 \%}=[2.48 ; 2.92]\right)$ und von universitären ExpertInnen mit 2.56 $\left(\mathrm{Cl}_{95 \%}=[2.29 ; 2.82]\right)$ und somit nicht signifikant positiv bewertet.

Bei der Forschungsplanung ist Methodenwissen notwendig, um die Datenerhebung methodenkonform umzusetzen. Besonders wichtig sei hier die Kenntnis der Eigenschaften guter Erhebungsinstrumente (QL5.B: 44, QN2.B: 43, QN1.B: 6, QL3.B: 6). So würden beispielsweise Interviewleitfäden zu sehr an der alltäglichen Kommunikationsweise ausgerichtet (QL4.B: 14) oder Fragebögen zu umfangreich gestaltet (QN3.B: 40, QL6.B: 46). Die zugehörigen Testaufgaben wurden von PraxisexpertInnen im Durchschnitt mit 3.19 ( Cl $_{95 \%}=[3.02$; 3.36]) besonders positiv, jedoch von universitären ExpertInnen mit 2.64 ( $\mathrm{Cl}_{95 \%=[2.36 ; 2.92])}$ nicht signifikant positiv bewertet. Bei der Datenauswertung und -interpretation ist Methodenwissen erforderlich, um sicherzustellen, dass alle notwendigen Auswertungsschritte ausgeführt werden. Das Auslassen von Auswertungsschritten wurde mehrfach als Defizit studentischer Forschungsarbeiten genannt (QL5.B: 46, QL6.B: 44, QN2.B: 41, QN1.B: 8-11). Neben der Kenntnis von Auswertungsschritten sei es zudem wichtig, dass die Studierenden wissen, wie Daten zu interpretieren sind. So würden den Studierenden bei der Dateninterpretation häufig Fehler unterlaufen (QL5.B: 42, QN1.B: 9). Die zugehörigen Testaufgaben wurden von

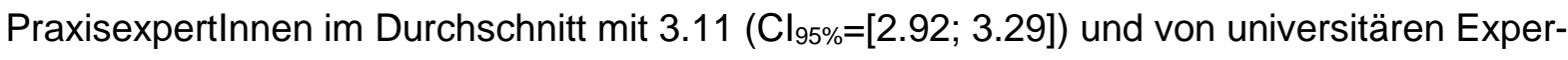
tInnen mit $2.83\left(\mathrm{Cl}_{95 \%}=[2.57 ; 3.10]\right)$ und somit in beiden Expertengruppen signifikant positiv bewertet.

\subsection{Methodologisches Wissen}


Das methodologische Wissen wurde besonders von ExpertInnen der qualitativen Methodentradition als bedeutsam beschrieben. Es bestimme die Perspektive auf den Forschungsgegenstand. Zentral sei dabei die Kenntnis der methodologischen Grundbegriffe: „Es muss klar sein, [...] dass man natürlich in dieser Tradition bestimmten Grundbegriffen folgt" (QL3.I: 325).

Neben den Grundbegriffen umfasst das methodologische Wissen zudem die Kenntnis der zu einer Methodologie passenden Erhebungs- und Auswertungsmethoden. Zudem sind die methodologischen Grundprinzipien (QL3.I: 503), die der Anwendung von Forschungsmethoden zugrunde liegen, Teil des methodologischen Wissens. Dies beinhaltet das „Wissen um die Unterschiedlichkeit qualitativer und quantitativer Forschungsprozesse“ (QL4.B: 2).

Zur Problemherleitung ist methodologisches Wissen notwendig, um Forschungsfrage und gewählte Methodologie aufeinander abstimmen zu können. Die Verwendung methodologischer Grundbegriffe in einer Forschungsfrage habe eine Implikation für den weiteren Forschungsprozess. In der quantitativen Methodentradition sei ein solcher Grundbegriff der „kausale Zusammenhang“ (QN3.B: 19), in der qualitativen Methodentradition wurden „handlungsleitende Orientierungen“ (QL5.B: 22), „soziale Wirklichkeit“ (QL6.B: 19) und „Interaktion“ (QL6.B: 20) genannt. Die zugehörigen Testaufgaben wurden von PraxisexpertInnen im Durchschnitt mit $3.01\left(\mathrm{Cl}_{95 \%}=[2.81 ; 3.21]\right)$ und von universitären ExpertInnen mit 2.89 $\left(\mathrm{Cl}_{95 \%}=[2.60 ; 3.18]\right)$ und somit signifikant positiv bewertet.

Bei der Forschungsplanung ist ebenfalls die Kenntnis methodologischer Grundbegriffe erforderlich. Beispiele für die Grundbegriffe seien in der quantitativen Methodentradition die „Hypothese, Falsifikation, Bewährung“ (QN2.B: 21), „Stichprobe, Operationalisierung, Variable, [...] Hilfs- oder Messtheorie“ (QN3.B: 19), sowie „probabilistische Schlussschemata“ und „Experiment“ (QN1.B: 30). In der qualitativen Methodentradition müssten vor allem die Begriffe „Kategorie, Kodierparadigma“ (QL6.B: 22) und „Rekonstruktion“ (QL4.B: 22) bekannt und verstanden sein. Zudem sei die Kenntnis der zu einer Methodologie passenden Erhebungs- und Auswertungsmethoden (QL5.B: 33, QN2.B: 30) erforderlich. Die zugehörigen Testaufgaben wurden von beiden Expertengruppen signifikant positiv bewertet: bei PraxisexpertInnen mit $3.06\left(\mathrm{Cl}_{95 \%}=[2.87 ; 3.25]\right)$, bei universitären ExpertInnen mit $3.17\left(\mathrm{Cl}_{95 \%=[2.88 \text {; }}\right.$ 3.45]).

Bei der Datenauswertung und -interpretation ist die Kenntnis der bei der Interpretation von Daten zu berücksichtigenden methodologischen Grundprinzipien („Analyseeinstellung“, QL3.I: 497) sowie die Kenntnis der Grenzen von Methodologien notwendig. Grundprinzipien seien die Berücksichtigung der von Interviewpartnern selbst hergestellten Kontexte (QL3.B: 8) oder das Verständnis von Signifikanz (QN2.B: 28). Typische Fehler seien die Überschätzung der Bedeutung von Signifikanz (QN3.B: 26) oder quantifizierende Aussagen bei qualita- 
tiven Methoden (QL6.B: 31). Die Testaufgaben wurden von beiden Expertengruppen signifikant positiv bewertet: von PraxisexpertInnen mit $3.26\left(\mathrm{Cl}_{95 \%}=[3.08 ; 3.45]\right)$, von universitären ExpertInnen mit $2.92\left(\mathrm{Cl}_{95 \%}=[2.64 ; 3.19]\right)$.

Tabelle 5: $\quad$ Übersicht über die Mittelwerte der Ratings nach Modellbestandteilen

\begin{tabular}{|c|c|c|c|c|}
\hline & & $\begin{array}{c}\text { Forschungsprozess- } \\
\text { wissen }\end{array}$ & Methodenwissen & $\begin{array}{c}\text { methodologisches } \\
\text { Wissen }\end{array}$ \\
\hline \multirow{2}{*}{$\begin{array}{l}\text { Problemherlei- } \\
\text { tung }\end{array}$} & $\mathrm{P}$ & 2.67 & 2.70 & 3.01 \\
\hline & $U$ & 2.75 & 2.56 & 2.89 \\
\hline \multirow{2}{*}{$\begin{array}{l}\text { Forschungspla- } \\
\text { nung }\end{array}$} & $\mathrm{P}$ & 3.06 & 3.19 & 3.06 \\
\hline & $U$ & 2.89 & 2.64 & 3.17 \\
\hline \multirow{2}{*}{$\begin{array}{l}\text { Datenauswer- } \\
\text { tung und -inter- } \\
\text { pretation }\end{array}$} & $\mathrm{P}$ & 3.08 & 3.11 & 3.26 \\
\hline & $U$ & 3.19 & 2.83 & 2.92 \\
\hline
\end{tabular}

Anmerkungen: $\mathrm{P}=$ Mittelwert der Praxisexpertenratings, $\mathrm{U}=$ Mittelwert der Ratings universitärer Experten

\section{Diskussion und Schlussfolgerungen}

\subsection{Zusammenfassung und Diskussion}

Im vorliegenden Beitrag wurde ein Modell von Forschungskompetenz in den Sozialwissenschaften induktiv auf Basis von Expertenaussagen entwickelt und ausdifferenziert. Das Modell unterscheidet drei zentrale Kompetenzdimensionen: (1) Forschungsprozesswissen, das eine planende und steuernde Funktion einnimmt, (2) Methodenwissen, das die korrekte Anwendung spezifischer Forschungsmethoden sicherstellen soll und (3) methodologisches Wissen, das den methodologischen Rahmen um die Forschungsmethoden bildet und die Verwendung von Grundbegriffen und -prinzipien steuert.

Obwohl die Kompetenzdimensionen separat eingeführt wurden, hängen sie eng zusammen, wobei das Forschungsprozesswissen eine zentrale Rolle einzunehmen scheint: Es greift auf das methodologische Wissen zurück, wenn bei der Formulierung der Forschungsfrage die Verwendung präziser Begriffe geprüft wird und auf das Methodenwissen, wenn der methodische Aufwand einer Forschungsfrage geprüft und die Zeitplanung vorgenommen wird. Aufgrund seiner steuernden Funktion ist anzunehmen, dass das Forschungsprozesswissen die zentrale Kompetenzdimension bildet, um die herum sich die anderen Kompetenzdimensionen gruppieren.

Auch das Methoden- und methodologische Wissen hängen eng zusammen. Werden beispielsweise Erhebungsinstrumente entwickelt oder Erhebungs- und Auswertungsmethoden ausgewählt, sollten diese zur gewählten methodologischen Ausrichtung passen. Auf Basis 
der Expertenaussagen lässt sich eine Differenzierung dieser beiden Kompetenzdimensionen vornehmen, insbesondere bei den qualitativen Methoden. Offen ist jedoch, ob dies auch bei Studierenden der Fall ist, die über weniger ausdifferenzierte Wissensstrukturen verfügen.

Neben den Kompetenzdimensionen berücksichtigt das hier entwickelte Modell drei als zentral identifizierte Forschungstätigkeiten: (1) die Problemherleitung, (2) die Forschungsplanung und (3) die Datenauswertung und -interpretation. Die identifizierten Kompetenzdimensionen und Forschungstätigkeiten wurden miteinander in Bezug gesetzt, so dass ausdifferenziert werden konnte, welche Wissensbereiche (personenspezifische Komponenten) bei welchen Forschungstätigkeiten (situationsspezifische Komponenten) zum Tragen kommen. Anhand der Ergebnisse der Expertenbefragung wurde deutlich, dass jede der Kompetenzdimensionen bei jeder der drei Forschungstätigkeiten erforderlich ist.

In einem Expertenrating wurden Testaufgaben, die auf Basis des Modells entwickelt wurden, von Praxis- und universitären ExpertInnen bewertet. Die Bewertungen der zugehörigen Testaufgaben lagen bei allen Kombinationen aus Kompetenzdimensionen und Forschungstätigkeiten und bei beiden Expertengruppen im Durchschnitt im oberen Bereich der Bewertungsskala. Das anhand weniger ExpertInnen entwickelte Kompetenzmodell hielt insofern auch der indirekten Beurteilung durch insgesamt 80 ExpertInnen stand.

Lediglich in einzelnen Bereichen konnte keine signifikant positive Bewertung festgestellt werden. Dies betraf vor allem die Testaufgaben zur Problemherleitung. So war beispielsweise erkennbar, dass die PraxisexpertInnen Testaufgaben zur Problemherleitung fast durchgängig als weniger bedeutsam beurteilten. Möglicherweise dominiert im beruflichen Alltag der PraxisexpertInnen die Auftragsforschung, bei der die Forschungsprobleme zumeist bereits vorgegeben sind. Aber auch bei universitären ExpertInnen zeigten sich hier vergleichsweise niedrige Bewertungen. Andererseits wies die Problemherleitung eine so hohe Relevanz für die interviewten ExpertInnen auf, dass sie viel Redezeit auf Schwierigkeiten der Studierenden bei diesem Forschungsschritt verwandten. Diese Diskrepanz könnte darauf deuten, dass die Testaufgaben zur Problemherleitung den Kern des Konstrukts nicht trafen.

\subsection{Limitationen}

Bei der Modellierung wurden vier Eingrenzungen vorgenommen: Erstens wurde in der vorliegenden Studie ein kognitiver Kompetenzbegriff zugrunde gelegt (Eraut, 2002) und affektiv-motivationale Voraussetzungen (Weinert, 2001b; Wessels, Rueß, Jenßen, Gess, \& Deicke, 2018) von forschungskompetentem Handeln sowie situationsspezifische Fertigkeiten (Blömeke, Gustafsson, \& Shavelson, 2015) nicht thematisiert. Das Zusammenspiel kognitiver und affektiv-motivationaler Facetten bei der Erklärung situationsspezifischer Fertigkeiten könnte zukünftig näher untersucht werden. 
Zweitens enthält das Modell bislang nur eine Auswahl an Forschungstätigkeiten. Zu prüfen wäre, wie eng Forschungskompetenz in der hier vorgenommenen Beschränkung mit Konstrukten zusammenhängt, die andere Forschungstätigkeiten fokussieren, wie die Rezeption und Anwendung von Forschungsergebnissen (Groß Ophoff et al., 2014).

Drittens wurden ExpertInnen aus der Psychologie erst im letzten Schritt einbezogen. Das Kompetenzmodell wurde ohne die psychologische Perspektive entwickelt, so dass etwa fraglich bleibt, ob in der Psychologie weitere Kompetenzdimensionen von Bedeutung sind. Dies schränkt die Generalisierbarkeit des Modells im Hinblick auf die Psychologie ein.

Viertens bleibt offen, ob die berufspraktische Relevanz des Modells auch aus Sicht anderer Tätigkeitsfelder als der hier betrachteten gegeben ist. So ist durchaus anzunehmen, dass etwa im Lehramt weitere Aspekte, wie die Einsicht in die Komplexität des Theorie-PraxisVerhältnisses (Meyer, 2003), zu berücksichtigen wären. Da die an der vorliegenden Studie beteiligten ExpertInnen nicht aus Lehramtsstudiengängen rekrutiert wurden, ist das Modell nicht für sie generalisierbar. Eine Ergänzungsstudie explizit für das Lehramtsstudium scheint insofern angebracht.

\subsection{Schlussfolgerungen und Ausblick}

Im Ergebnis wird mit dem vorliegenden Beitrag erstmals ein innerhalb der Sozialwissenschaften fachübergreifendes Modell der kognitiven Facette von Forschungskompetenz vorgelegt. Das Modell geht in seinem Differenzierungsgrad über bestehende Modelle hinaus, die zumeist auf Performanzaussagen beruhen, d.h. lediglich aufzählen, welche Forschungstätigkeiten erfolgreich zu absolvieren sind. In dem neu entwickelten Modell wird demgegenüber dargestellt, welche Wissensbereiche welchen Forschungstätigkeiten zugrunde liegen. Darüber hinaus werden auch inhaltlich neue Akzente gesetzt. So wurde mit dem Forschungsprozesswissen eine zuvor weitgehend unbeachtete Kompetenzdimension identifiziert. Denkbar wäre nun eine Berücksichtigung des Modells bei der Gestaltung oder Überprüfung von Studienordnungen. Zu vermuten ist auf Basis einer Curriculumanalyse (Rueß, Gess, \& Deicke, 2016) etwa, dass das Forschungsprozesswissen in einigen Studiengängen kaum adressiert wird. Ebenso wäre ein Abgleich mit Modellen wissenschaftspropädeutischer Bildung von Interesse, da bereits die schulische Oberstufe an wissenschaftliches Denken heranführen soll (Trautwein \& Lüdtke, 2004).

Mit den positiv bewerteten Testaufgaben besteht nun zudem eine geeignete Grundlage, um die sozialwissenschaftliche Forschungskompetenz von Studierenden standardisiert und mit größeren Fallzahlen zu erheben. Für die Inhaltsvalidität des Modells und Testinstruments wurde durch das stark auf Expertenaussagen und -urteilen aufbauende Vorgehen ein solides Fundament bereitet (Jenßen et al., 2015). Weitere Validierungsarbeiten sind gemäß der Standards für Validierung (AERA, APA, \& NMCE, 2014) erforderlich. Erste Analysen deuten 
darauf hin, dass die Testwerte ein erlernbares, sozialwissenschaftliches und forschungsbezogenes Konstrukt wiedergeben (Gess, Geiger, \& Ziegler, 2018). Offen ist, ob die Testwerte extern bewertete Forschungsleistungen und Berufserfolg in wissenskonstruierenden Berufen vorhersagen können.

Mithilfe des Testinstruments könnten zukünftig Entwicklungsprozesse während des Studiums, individuelle Prädiktoren sowie Effekte von Ausbildungsangeboten ermittelt werden: Welche Arten von Lehrveranstaltungen sind zur Vermittlung von Forschungskompetenz geeignet, eher dimensions- und tätigkeitsbezogene oder integrative Veranstaltungen? Zudem können einzelne Kompetenzdimensionen fokussiert werden. Hier bietet sich besonders das im vorliegenden Beitrag als zentral identifizierte Forschungsprozesswissen an. Sind hierfür aufwendige Formate wie Forschendes Lernen erforderlich? Insgesamt stellen sich also zahlreiche reizvolle Forschungsaufgaben, mit denen eine kompetenzorientierte Auseinandersetzung über die Forschungsausbildung begonnen werden kann. 
Literatur

Abreu, B. C., Peloquin, S. M., \& Ottenbacher, K. (1997). Competence in scientific inquiry and research. The American Journal of Occupational Therapy, 52(9), 751-759.

AERA, APA, \& NMCE. (2014). Standards for educational \& psychological testing. Washington, DC: American Educational Research Association.

Blömeke, S., Gustafsson, J.-E. E., \& Shavelson, R. J. (2015). Beyond dichotomies: Competence viewed as a continuum. Zeitschrift für Psychologie / Journal of Psychology, 223(1), 3-13. http://doi.org/10.1027/2151-2604/a000194

Charmaz, K. (2014). Constructing Grounded Theory (2. Aufl.). London: SAGE.

Cohen, J. (1960). A coefficient of agreement for nominal scales. Educational and Psychological Measurement, 20(1), 37-46.

Cronbach, L. J. (1951). Coefficient alpha and the internal structure of tests. Psychometrika, 16(3), 297-334.

Deutsche Gesellschaft für Evaluation. (2008). Empfehlungen für die Aus- und Weiterbildung in der Evaluation. Abgerufen 5. Januar 2018, von

http://www.degeval.de/fileadmin/Publikationen/Publikationen_Homepage/DeGEval__Empfehlungen_Aus-_und_Weiterbildung.pdf

DFG. (2017). DFG-Fachsystematik der Wissenschaftsbereiche, Fachgebiete, Fachkollegien und Fächer für die Amtsperiode 2016-2019. Abgerufen 2. Mai 2018, von

http://www.dfg.de/download/pdf/dfg_im_profil/gremien/fachkollegien/amtsperiode_2016 _2019/fachsystematik_2016-2019_de_grafik.pdf

Earley, M. A. (2014). A synthesis of the literature on research methods education. Teaching in Higher Education, 19(3), 242-253. http://doi.org/10.1080/13562517.2013.860105

Eraut, M. (2002). Developing professional knowledge and competence. London, New York: Routledge.

Esser, G., Groeger, W., Munz, D., Lehndorfer, P., \& Schwartz, H.-J. (2010). Reform der Psychotherapeutenausbildung. Abgerufen 5. Januar 2018, von http://www.bptk.de/uploads/media/20100216_bptk_konkretisierung_eckpunkte_pt_ausbi ldung.pdf

European Commission. (2013). Supporting teacher competence development for better learning outcomes. Abgerufen 1. Juli 2016, von http://ec.europa.eu/education/policy/school/doc/teachercomp_en.pdf

Gess, C., Geiger, C., \& Ziegler, M. (2018). Social-scientific research competency: Validation of test score interpretations for evaluative purposes in higher education. European Journal of Psychological Assessment.

Groß Ophoff, J., Schladitz, S., Lohrmann, K., \& Wirtz, M. (2014). Evidenzorientierung in bildungswissenschaftlichen Studiengängen: Entwicklung eines Strukturmodells zur Forschungskompetenz. In K. Drossel (Hrsg.), Empirische Bildungsforschung und evidenzbasierte Reformen im Bildungswesen (S. 251-275). Münster: Waxmann.

Ivanenko, N. A., Mustafina, G. M., Sagitova, V. R., Akhmetzyanov, I. G., Khazratova, F. V., Salakhova, I. T., \& Mokeyeva, E. V. (2015). Basic components of developing teachers' research competence as a condition to improve their competitiveness. Review of European Studies, 7(4), 221-227.

Jenßen, L., Dunekacke, S., \& Blömeke, S. (2015). Qualitätssicherung in der Kompetenzforschung: Standards für den Nachweis von Validität in Testentwicklung und Veröffentlichungspraxis. Zeitschrift für Pädagogik (Beiheft), 61, 11-30. 
Kilburn, D., Nind, M., \& Wiles, R. (2014). Learning as researchers and teachers: The development of a pedagogical culture for social science research methods? British Journal of Educational Studies, 62(2), 191-207. http://doi.org/10.1080/00071005.2014.918576

Klieme, E., \& Leutner, D. (2006). Kompetenzmodelle zur Erfassung individueller Lernergebnisse und zur Bilanzierung von Bildungsprozessen. Beschreibung eines neu eingrichteten Schwerpunktprogramms der DFG. Zeitschrift für Pädagogik, 52(6), 876903.

Kuckartz, U. (2014). Mixed Methods: Methodologie, Forschungsdesigns und Analyseverfahren. Springer.

Kultusministerkonferenz. (2014). Standards für die Lehrerbildung: Bildungswissenschaften. Bonn: Sekretariat der Kultusministerkonferenz. Abgerufen 5. Januar 2018, von http://www.kmk.org/fileadmin/Dateien/veroeffentlichungen_beschluesse/2004/2004_12_ 16-Standards-Lehrerbildung.pdf

Mayring, P. (2010). Qualitative Inhaltsanalyse. In G. Mey \& K. Mruck (Hrsg.), Handbuch Qualitative Forschung in der Psychologie (S. 601-613). Wiesbaden: VS Verlag für Sozialwissenschaften.

McDonald, R. P. (1999). Test theory: a unified treatment. Hillsdale: Erlbaum.

Meuser, M., \& Nagel, U. (2005). ExpertInneninterviews - vielfach erprobt, wenig bedacht. In A. Bogner, B. Littig, \& W. Menz (Hrsg.), Experteninterviews - Theorien, Methoden, Anwendungen (S. 71-94). Wiesbaden: VS Verlag für Sozialwissenschaften / GWV Fachverlage $\mathrm{GmbH}$.

Meyer, H. (2003). Skizze eines Stufenmodells zur Analyse von Forschungskompetenz. In A. Obolenski \& H. Meyer (Hrsg.), Forschendes Lernen - Theorie und Praxis einer professionellen LehrerInnenausbildung (S. 99-116). Bad Heilbrunn: Klinkhardt.

Norman, G. (2010). Likert scales, levels of measurement and the „laws" of statistics. Advances in Health Sciences Education, 15(5), 625-632.

Rueß, J., Gess, C., \& Deicke, W. (2016). Forschendes Lernen und forschungsbezogene Lehre - empirisch gestützte Systematisierung des Forschungsbezugs hochschulischer Lehre. Zeitschrift für Hochschulentwicklung, 11(2), 23-44.

Thiel, F., \& Böttcher, F. (2014). Modellierung fächerübergreifender Forschungskompetenzen. In B. Berendt, A. Fleischmann, N. Schaper, B. Szczyrba, \& J. Wildt (Hrsg.), Neues Handbuch Hochschullehre (S. 109-125). Berlin: Raabe.

Trautwein, U., \& Lüdtke, O. (2004). Aspekte von Wissenschaftspropädeutik und Studierfähigkeit. In O. Köller, R. Watermann, U. Trautwein, \& O. Lüdtke (Hrsg.), Wege zur Hochschulreife in Baden-Württemberg: TOSCA - Eine Untersuchung an allgemein bildenden und beruflichen Gymnasien (S. 327-366). Wiesbaden: VS Verlag für Sozialwissenschaften.

Weinert, F. E. (2001a). Concept of competence: a conceptual clarification. In D. S. Rychen \& L. H. Salganik (Hrsg.), Defining and Selecting Key Competencies (S. 45-66). Seattle: Hogrefe.

Weinert, F. E. (2001b). Vergleichende Leistungsmessung in Schulen: Eine umstrittene Selbstverständlichkeit. In F. E. Weinert (Hrsg.), Leistungsmessungen in Schulen (S. 1731). Weinheim: Beltz.

Wessels, I., Rueß, J., Jenßen, L., Gess, C., \& Deicke, W. (2018). Beyond Cognition: Experts' Views on Affective-Motivational Research Dispositions in the Social Sciences. Frontiers in Psychology, 9(1300).

Wester, K. L., \& Borders, L. D. (2014). Research competencies in counseling: a Delphi study. 
Journal of Counseling \& Development, 92(4), 447-458. http://doi.org/10.1002/j.15566676.2014.00171.x

Wild, E., \& Esdar, W. (2016). Beiträge der Pädagogischen Psychologie zur Wissenschaftspolitik. In D. Simon (Hrsg.), Handbuch Wissenschaftspolitik (S. 191-205). Wiesbaden: Springer.

Wissenschaftsrat. (2006). Empfehlungen zur künftigen Rolle der Universitäten im Wissenschaftssystem. Berlin. Abgerufen 5. Januar 2018, von https://www.wissenschaftsrat.de/download/archiv/7067-06.pdf

Wissenschaftsrat. (2008). Empfehlungen zur Qualitätsverbesserung von Lehre und Studium. Berlin. Abgerufen 5. Januar 2018, von https://www.wissenschaftsrat.de/download/archiv/8639-08.pdf

Zlatkin-Troitschanskaia, O., Shavelson, R. J., \& Kuhn, C. (2015). The international state of research on measurement of competency in higher education. Studies in Higher Education, 40(3), 393-411. 